\title{
Effects of a Behavioral Program on Exercise Adherence and Exercise Self-Efficacy in Community-Dwelling Older Persons
}

\author{
Azliyana Azizan, Maria Justine, and Chua Siew Kuan \\ Faculty of Health Sciences, Puncak Alam Campus, Universiti Teknologi MARA (UiTM), 42300 Puncak Alam, Selangor, Malaysia \\ Correspondence should be addressed to Maria Justine; maria205@salam.uitm.edu.my
}

Received 28 August 2013; Revised 10 November 2013; Accepted 1 December 2013

Academic Editor: T. Kostka

Copyright ( 2013 Azliyana Azizan et al. This is an open access article distributed under the Creative Commons Attribution License, which permits unrestricted use, distribution, and reproduction in any medium, provided the original work is properly cited.

\begin{abstract}
Background. This study determines the effects of a behavioral program on exercise adherence (step counts) and level of exercise self-efficacy (ESE) in community-dwelling older persons. Methods. Sixty-three participants (age $=63.8 \pm 4.5$ years) were enrolled in this controlled quasi-experimental study. They were divided into 3 groups: (1) EBG performed a 6-week exercise intervention followed by a 5-week behavioral program, (2) EG performed exercise intervention similar to EBG, and (3) control group (CG) did not receive any interventions. Step counts were measured based on the scores recorded by a pedometer while ESE was measured by a self-reported ESE scale. Results. Data analysis showed significant differences due to time effect $(F(1,2)=39.884, P<0.01$, and $\eta=.399)$; time and group interactions $(F(2,60)=112.683, P<0.01$, and $\eta=.790)$; and between-group effect $(F(2,60)=12.524$, $P<0.01$, and $\eta=.295)$ for step counts. As for ESE, significant differences were also found for time effect $(F(2,4)=66.628$, $P<0.05$, and $\eta=.526)$; time and group interactions $(F(2,60)=4.562, P=0.014$, and $\eta=.132)$; and between-group effect $(F(2,60)=13.632, P<0.05$, and $\eta=.312)$. EBG presented with significantly higher mean changes for both step counts and ESE compared to other groups (all $P<0.05$ ). Conclusion. This study suggests that the addition of a behavioral program is superior as compared to exercising alone on increasing exercise adherence and level of self-efficacy in older persons.
\end{abstract}

\section{Introduction}

Purposeful exercise and increased participation in physical activity are among the most important components of lifestyle, especially for older persons [1]. Participation in these activities is considered an important behavior that may yield benefits especially on maintaining the physical and psychological well-being [2-5]. A published review on physical activity and aging highlighted that older persons who actively participated in a long term exercise program appear to have a good quality of life [6]. Regrettably, majority of old people around the world lead a sedentary lifestyle with very little knowledge regarding the importance of physical activity. As highlighted by Irvine et al. [7], almost 39\% older persons do not meet recommended physical activity guidelines and 33\% also reported no leisure-time physical activity. This meant that a portion of older people did not meet the recommended exercise program suggested by the American College of Sports Medicine [8]. An exercise program for older persons must include a multicomponent training, namely, endurance, flexibility, strengthening, and balance [8]. Older persons are encouraged to perform moderate intensity exercise 5 days per week with a minimum of 30 minutes per session. This recommendation aims to target all the major physiological systems that are functionally declining due to the aging process. For instance, a regular resistance training program may induce muscle hypertrophy by altering the expression of myosin heavy isoforms, [9], while aerobic exercise improves blood pressure and decreases the risk of coronary artery disease [10]. Balance training also helps in reducing fear of falls and improving dynamic balance [11]. Thus, it is clear that each training component may contribute to better physical health status.

One of the major issues related to participation in an exercise program is the compliance or adherence to such program that may be influenced or determined by numerous factors. A previous study has identified these determinants and grouped them into two main categories: either increase adherence to exercise (motivators) or decrease adherence to exercise (barriers) [12]. However, most studies consistently 
found that self-efficacy was the strongest predictor of exercise adherence [13-15]. Self-efficacy can be defined as perceived capability and confidence, specific to a particular domain of behavior [16]. In older persons, self-efficacy predicts exercise adherence [17], and this can be used to control and motivate them to engage in physical activity and exercise for as long as possible. Thus, this indicates a need to develop a theory-based physical activity and exercise intervention that can be linked to the concept of long term exercise adherence. This is vital since exercising has to be an ongoing activity in order to gain its immediate and long term positive effects.

Therefore, this study was carried out to develop interest and fostering motivation to help increase daily participation in physical activity among older persons living in the community through theory-based behavioral approaches. While the behavioral interventions have been revealed to provide positive outcomes in increasing level of physical activity [18], most, however, were applied and tested only for chronic illnesses such as depression [19], chronic low back pain [20], older persons with fear of falls [21], and diabetes type-2 [22]. There are a limited number of studies that have focused on the perception and behavior towards exercise, specifically among older persons residing in the community. Community-dwelling older persons are the main target against those institutionalized because they may differ in terms of their level of exercise adherence. One possible reason could be due to their other commitments, such as caring for grandchildren, running businesses, weekly meetings, and spiritual and voluntary activities. As such, this study was designed to determine the effects of a behavioral program intervention following a short term introduction to exercise program on exercise adherence and exercise selfefficacy. An exercise program was implemented prior to the initiation of the behavioral program to introduce the different components of exercise that are safe and effective for senior people. In this study, a pedometer was used to verify the step counts as a measure of level of physical activity $[23,24]$. As is commonly known, a pedometer can detect steps accurately as an indication of volume of physical activity $[25,26]$. In addition, the number or visual indicators on the pedometer can provide feedback in order to motivate people to increase their level of physical activity [26].

\section{Methods}

2.1. Study Design. A controlled quasi-experimental study was conducted over a period of 24 weeks. The participants were divided into three groups; (1) exercise and behavioral programs (EBG), (2) exercise program only (EG), and (3) control group (CG). The EBG received a six week exercise program adapted from a previous study [27], with increasing frequency and intensity. The exercise training was also added with a locomotor training such as dancing and tai chi movements. The termination of exercise was then followed by a behavioral program for another five weeks. The EG received the same exercise protocol as in group EBG for six weeks but without the behavioral program. While the control group (CG) was not given any interventions but instructed to continue their usual activities, the protocol for this study was approved by the Ethics Committee of the University. Each participant was assured of confidentiality and the option to withdraw at any time without penalty.

2.2. Study Setting and Participants. Ninety older persons aged above 60 years were recruited from three different villages, around Selangor Malaysia. Each village was allocated with only one type of intervention in order to avoid communications among the participants (Village 1 = EBG; Village 2 = EG; Village $3=\mathrm{CG}$ ). Prior to the initiation of the intervention, all participants were screened for eligibility. Participants were included based on the following criteria; (1) aged 60 years old and above, (2) have no cognitive impairments (for those with MMSE score, <24 were excluded) [28], (3) independent in activities of daily living, (4) able to understand English/Malay, and (5) resided in the study setting. Subjects presented with chronic musculoskeletal problem, dementia or neurological disorders, severe hearing and visual impairment, and having an inability to walk were excluded from this study. Seventy-three older persons $(E B G=25$, $\mathrm{EG}=25$, and $\mathrm{CG}=23$ ) who fulfilled the inclusion criteria were included in this study. At the end of the study, a few participants dropped out from the study, with remaining 18 in EBG, 23 in EG, and 22 in CG. The participants who were dropped out cited reasons such as no transportation to the study site, time constrains, and weather problems.

Participants from three different villages were chosen in order to reduce the effects of behavioral influence to other participants who may be motivated to perform the intervention that was not assigned to them. These villages were chosen based on their homogeneity in terms of level of education, ethnicity, previous nature of jobs, basic infrastructures (such as community halls and community clinic), and level of physical activities. At the time of the implementation of this study, none of the participants were carrying out any formal or informal exercise programs. As such, they were assumed to present with the same level of physical activity at baseline. We considered homogeneity among participants as an important factor to reduce the biasness in terms of baseline performance of physical activity. This is supported by Schöllgen et al. [29] that argued participants with significant differences in demographic status may have different levels of physical and psychological health especially in relation to the level of education and vice versa. Thus, this situation may affect their behavior towards exercise participation.

2.2.1. The Exercise Program. This exercise program was designed based on the aspects of fitness for older persons and comprised of cardiovascular endurance, muscle strength, balance, flexibility, and locomotor training. The main target was to involve all major muscle groups of the body. The intensity and the volume of exercise were increased gradually to prevent any adverse effects of exercise. The exercise program consisted of 18 sessions with each lasted for about 60 minutes, carried out over a period of six weeks, three sessions a week. The exercises were conducted in sitting positions 
in the first two weeks (1st phase) then followed by standing positions in the last 4 weeks (2nd phase).

Each session consisted of 8 to 10 minutes of stretches for the major muscle group (pectoral, latissimus dorsi, cervical paravertebral, and posterior and anterior thigh muscles); 20 minutes of aerobic training; 20 minutes of strength training (with load determined by theraband and body weight); 10 minutes of locomotor, coordination, and agility and flexibility exercises. All sessions were conducted group based, supervised by the researcher and assisted by trained physiotherapists. The sessions were also incorporated with music, to keep the participants motivated, as suggested in a meta-analysis study [30]. A weekly meeting was held among the research members to ensure consistency of the sequence and pattern of the exercises for all participants.

2.2.2. The Behavioral Program. The behavioral program was designed based on the "stages of change" model, which is one of the components in the "Transtheoretical model of behavior change" (TTM). This model was originally postulated by Prochaska et al. [31]. Several studies have found that the stages of change in TTM can be predictive of behavior change in older persons. For example, TTM was used for changing and implementing home safety modifications with communitydwelling older persons [32]. Another example includes the use of TTM for physical activity in older persons with type 2 diabetes and cardiovascular disease [33].

The aim of this behavioral program was to develop effective intervention to promote health behavior change, especially towards exercise participations. In essence, 5 modules were developed based on the 5 stages of change as postulated in TTM. For each stage of change, different behavioral strategies were implemented so that the older persons are motivated and may progress to the next stage.

The first stage is known as "precontemplation," where, at this stage, older persons do not intend to take action within the next 6 months. They may be unaware of the benefits of changing behavior and thus may produce negative thoughts about it. They may focus more on the cons of behavior changes compared to the pros. Next, the second stage is "contemplation." At this stage, older persons may intend to start healthy behavior in the future and may come to realize their problematic behavior. They may also consider the pros and cons of changing the behavior if it takes place, with equal emphasis placed on both. Even though they may recognize their problematic behavior, most may feel hesitant towards changing their behavior. This stage, however, will be followed by "preparation stage," in which older persons are ready to take action within the next 30 days. They start to take small steps towards the behavior change, and they believe changing their behavior can lead to a healthier life. In the fourth stage, the "action stage," older persons have recently changed their behavior and intend to keep moving forward with that behavior change. In this stage, older persons may modify their problematic behavior and may acquire new healthy behavior. The final part of the model will then be concluded by the "maintenance stage," where older persons sustained their behavior change for a while or for the next 6 months, in order to go forward.

In this study, however, we assumed that all participants were in the precontemplation stage. This is to reduce overestimation, simply because of the complexity of the behavior itself towards exercise and physical activity [34]. The strategies used in this study were presented by the researcher and trained physiotherapists through powerpoint presentations, face-to-face interview, and counseling sessions. Table 1 shows the protocol for the behavioral program used in this study, which was adapted from Jones and Rose [35] and Burbank et al. [36].

2.3. Study Outcome Measures. The primary outcome of this study is the level of physical activity also known as exercise adherence (steps count). The physical activity was measured objectively using a pedometer (Omron HJ-203; Omron Healthcare UK Ltd.). The pedometers were used for a period of 12 weeks, beginning at 12 th week until 24 th week after interventions. All records regarding the total daily step counts were documented in a pedometer sheet. The pedometer recorded step counts during participant's waking hours for seven days a week because it has a memory recall for up to seven days, allowing the research assistant to record any changes and recover the step counts in cases when participants failed to accurately record their step counts in the sheets [37]. The participants were asked to reach a target of 10,000 steps per day as recommended by the Ministry of Health, Malaysia, to stimulate people to take more frequent walks and carry out fitness activities [38]. Each participant wore the pedometer all day except during sleeping, bathing, and exercise program. The pedometer was positioned at the waist, in midline with the right middle thigh [39]. A research assistant who was blinded to the previous assessments and group allocation recorded participants' weekly step counts based on their own recording sheets.

The secondary outcome measure is the exercise selfefficacy which was measured by the exercise self-efficacy (ESE) scale [39]. The ESE scale is a self-reported questionnaire that contained nine items. The original English version of the ESE scale questionnaire was translated into Malay language (Bahasa Malaysia) based on the international guidelines [40]. The scales in the questionnaire measured the level of confidence on exercise performance started from 0 (not confident) to 5 (moderately confident) and up to 10 (very confident). Participants were instructed to rate their level of confidence on their ability to perform an exercise or walk for 20 minutes three times per week. The scale was scored by calculating the sum of the numerical ratings for each response which was divided by the number of responses.

2.4. Data Analysis. Changes in the number of steps and level of exercise self-efficacy were the main outcomes of this study. Results were reported as means $(\mathrm{m})$ and standard deviations (SD). A one-way ANOVA and $3 \times 2$ Chi-square tests were used to determine the baseline demographic differences between groups. The analysis of covariance was conducted to determine the presence of confounding variables at baselines 
TABLE 1: The behavioral intervention protocol.

\begin{tabular}{l}
\hline Activities \\
Week 1. Precontemplation \\
Goal: increase awareness of the need for change \\
Change process: consciousness raising, dramatic relief, and environmental reevaluation \\
Strategies: (1) encourage thinking about change \\
(2) discuss feelings and perceptions of exercise \\
(3) give personalized feedback about the risk of sedentary lifestyle and low exercise tolerance \\
(4) personalize information about the benefits of initiating an exercise program and how \\
inactivity affects role implementation and social activities
\end{tabular}

Week 2. Contemplation

Goal: motivate and increase confidence in the ability to change

Change process: consciousness raising, self-reevaluation, social liberation, self-liberation, and help participant to set realistic expectations reinforced by small success

Strategies: (1) continue explaining regarding personal risk and benefits about exercising

(2) decrease the barrier to exercise

(3) help them to make a definite commitment to change and goal setting

(i) Barriers and motivators worksheet was provided

(ii) Encourage participants to replace unhelpful thoughts with more productive thought into a "Positive Self Talk" sessions.

Week 3. Preparation

Goal: negotiate a plan for exercise

Change process: self-reevaluation, helping relationship, self-liberation, and help participant to set realistic expectations reinforced by small success

Strategies: (1) create a new self-image as an exerciser

(2) make a public commitment to exercise

(3) identify alternative for an exercise at home, individually or in a group.

(4) facilitate exercise efficacy involving support from others such as spouse and children

(5) encourage a component of behavioral change where they can experience success

(i) Barriers and motivators worksheet was provided

(ii) Use "Behavioral Contract and Presentation entitled "How to handle stress"' by the researcher.

Week. 4 Action

Goal: reaffirm commitment to exercise and implement the plan

Change process: reinforcement management, helping relationship, counter-conditioning, and stimulus control

Strategies: (1) talk on positive reinforcement with rewards/incentives and "role model" for the other group

(2) mobilizing social support to participate in the process or reinforce the process

(3) introduce additional exercise and correct techniques during exercising through behavioral manual given to each of the participants

(4) exercise log book and a group task

Week 5. Maintenance

Goal: problem solving

Change process: counter-conditioning, helping relationship, and reinforcement management

Strategies: (1) plan for resisting temptation to skip exercise sessions

(2) join an exercise group

(3) a meaningful reward for the successful exerciser

(4) behavioral manual

(i) All strategies were done through powerpoint presentations, face-to-face interview, and counseling, supervised by the researcher and trained physiotherapists.

(ii) Every Monday and Wednesday (30 min) for each week. 
TABLE 2: Characteristics of study participants $(n=63)$ at baseline.

\begin{tabular}{|c|c|c|c|c|c|c|c|}
\hline \multirow{2}{*}{ Characteristics } & \multicolumn{2}{|c|}{ EBG Group $(n=18)$} & \multicolumn{2}{|c|}{ EG Group $(n=23)$} & \multicolumn{2}{|c|}{ CG Group $(n=22)$} & \multirow{2}{*}{ Sig } \\
\hline & $n(\%)$ & $\mathrm{m} \pm \mathrm{SD}$ & $n(\%)$ & $\mathrm{m} \pm \mathrm{SD}$ & $n(\%)$ & $\mathrm{m} \pm \mathrm{SD}$ & \\
\hline Age (years) & & $66.1(6.21)$ & & $63.5(3.39)$ & & $62.3(3.07)$ & $\begin{array}{c}0.025^{*} \\
\left(\mathrm{EBG}-\mathrm{CG}^{*}\right)\end{array}$ \\
\hline \multicolumn{8}{|l|}{ Gender } \\
\hline Female & $8(44.4)$ & & $12(54.5)$ & & $15(65.2)$ & & \multirow{2}{*}{0.411} \\
\hline Male & $10(55.6)$ & & $10(45.5)$ & & $8(38.4)$ & & \\
\hline Body weight (kg) & & $59.82(3.33)$ & & $65.89(2.16)$ & & $64.187(1.66)$ & 0.202 \\
\hline Height (m) & & $1.53(0.03)$ & & $1.56(0.02)$ & & $1.57(0.02)$ & 0.521 \\
\hline BMI $\left(\mathrm{kg} / \mathrm{m}^{2}\right)$ & & $25.83(1.58)$ & & $27.05(0.65)$ & & $26.27(0.761)$ & 0.692 \\
\hline
\end{tabular}

Note. * Significant at $P<0.05$.

TABLE 3: Number of step counts for three groups across two point intervals.

\begin{tabular}{|c|c|c|c|c|c|c|c|c|c|c|}
\hline \multirow{2}{*}{ Time period } & \multicolumn{3}{|c|}{ EBG group } & \multicolumn{3}{|c|}{ EG group } & \multicolumn{3}{|c|}{ CG group } & \multirow{2}{*}{ Sig } \\
\hline & $n$ & Mean & SD & $n$ & Mean & SD & $n$ & Mean & $\mathrm{SD}$ & \\
\hline Baseline & 18 & 1491.28 & 220.134 & 23 & 1737.18 & 225.117 & 22 & 1627.09 & 183.688 & 0.724 \\
\hline 12th week & 18 & 48495.19 & 7884.38 & 23 & 12708.10 & 11144.67 & 22 & 6905.41 & 1609.12 & $0.00^{* *}$ \\
\hline 24 th week & 18 & 73883.75 & 15612.10 & 23 & 23352.06 & 27460.122 & 22 & 7982.46 & 3626.373 & $0.00^{* *}$ \\
\hline
\end{tabular}

Note. ${ }^{* *} P<0.01$.

for all groups. The repeated measures analysis of variance (ANOVA) was used to determine whether there were time factor effects, group effects, and interaction between the two factors (group and time) for all groups. An alpha level of $P<0.05$ was considered significant for all statistical tests.

\section{Results}

The final sample consisted of 63 participants (18 in EBG, 23 in EG, and 22 in CG group). The mean age of subjects was 63.8 ( $\mathrm{SD}=4.5$ years). The mean height, body weight, and $\mathrm{BMI}$ were $1.55(\mathrm{SD}=0.12 \mathrm{~m}), 63.53 \mathrm{~kg}(\mathrm{SD}=10.87 \mathrm{~kg})$, and $26.42 \mathrm{~kg} / \mathrm{m}^{2}\left(\mathrm{SD}=4.52 \mathrm{~kg} / \mathrm{m}^{2}\right)$, respectively. The baseline characteristics of study participants are shown in Table 2. There were no significant differences between the groups on demographic characteristics except for age variable, in which the significant difference was found between EBG and CG $(P<0.05)$.

The ANCOVA for age as the covariate was conducted for both number of steps and level of ESE at baseline. The results revealed that age was not a confounding factor for the number of steps (homogeneity of slopes, $F=0.131$; $P=0.877$. ANCOVA, $F=0.013 ; P=0.910)$ and level of ESE (homogeneity of slopes, $F=3.042 ; P=0.087$. ANCOVA, $F=$ $1.103 ; P=0.298$ ) at baseline. Therefore, further analysis using the repeated measures ANOVA was conducted to determine the effects of the intervention.

Table 3 shows that EBG attained the highest mean for number of steps at week 12 and week 24 compared to EG and CG. The repeated measures ANOVA showed significant differences in the number of steps at week 12 and up to 24 weeks due to the effect of time $(F(1,2)=39.884, P<0.01$, $\eta=.399)$; time and group interactions, $(F(2,60)=112.683$,
$P<0.01, \eta=.790)$; and between-group effect $(F(2,60)=$ $12.524, P<0.01, \eta=.295)$. The post hoc comparison using the Bonferroni test for mean difference between the three groups in the number of step counts showed a significant mean difference $(\mathrm{EBG}$ and $\mathrm{EG}=43159.38$; $\mathrm{EBG}$ and $\mathrm{CG}=$ 53725.53; EG and CG $=10586.15)$ with $P$ value $<0.05$.

Table 4 shows that the EBG scored the highest mean for ESE at week 24. Baseline level of ESE was not statistically significant different between the EBG, EG, and CG $(P>$ 0.05). The repeated measures ANOVA showed significant differences in the level of ESE due to the effect of time $(F(2,4)=66.628, P<0.05, \eta=.526)$; time and group interactions $(F(2,60)=4.562, P=0.014, \eta=.132)$; and between-group effects $(F(2,60)=13.632, P<0.05$, $\eta=.312$ ). However, the post hoc comparison using the Bonferroni test for mean difference between the three groups for ESE scores showed only significant difference between EBG and CG (10.90, $P<0.05)$. Further analysis showed that ESE score at week 12 was significantly different only between EBG and CG, while, at week 24, ESE differed significantly between EBG and EG as well as between EBG and CG (All, $P=0.01)$.

\section{Discussion}

The primary objective of this study was to determine the effects of a behavioral program following exercise participation on level of physical activity which was measured by the number of step counts recorded by a pedometer. The secondary objective was to determine the level of exercise self-efficacy (ESE) scores. The results obtained from this study are consistent with those of other meta-analysis studies of older persons that suggested behavior strategies were effective 
TABLE 4: Exercise self-efficacy scores for three groups across three time periods.

\begin{tabular}{lcccccccccc}
\hline \multirow{2}{*}{ Time period } & \multicolumn{3}{c}{ EBG group } & \multicolumn{3}{c}{ EG group } & \multicolumn{3}{c}{ CG group } \\
& $n$ & Mean & SD & $n$ & Mean & SD & $n$ & Mean & SD & 16.31 \\
Baseline & 18 & 35.00 & 14.65 & 23 & 39.09 & 19.00 & 22 & 38.70 & 0.698 \\
Week 12 & 18 & 53.33 & 10.29 & 23 & 48.86 & 11.332 & 22 & 41.30 & 14.24 & $0.009^{*}$ \\
Week 24 & 18 & 73.83 & 8.459 & 23 & 52.73 & 13.950 & 22 & 49.48 & 16.35 & $0.000^{*}$ \\
\hline
\end{tabular}

Note. ${ }^{*}$ Significant at $P<0.05$.

in changing behavior towards physical activity [41, 42]. There was a significant difference in the level of physical activity among the three groups. The EBG scored the highest mean average for the level of physical activity followed by the EG and CG.

The increased level of physical activity in the EBG indicates that the intervention may be effective in increasing participation in physical activity. However, both EG and CG recorded little difference in the number of steps. This is due to the fact that both did not receive any behavioral programs.

Participants in EBG showed a continuous increase in the number of steps which could largely be due to the agreement made by participants to comply with the behavioral contract, in which they were required to achieve a certain number of steps each week (e.g., 10,000 steps per day). This is advantageous as the pedometer can act as a visual feedback for older persons. The number of steps can be monitored personally at any time and users will be able to gauge the amount of steps needed to satisfy each individual's requirement. In addition, the behavioral contract could further motivate participants to adhere to the exercise activities. In contrast, without the behavioral contract, participants in the EG and CG lack the motivation needed to reach a higher number of steps.

As highlighted in a meta-analysis study, the use of a pedometer has a reasonable and constructive effect on the increase of physical activity across age groups, including older persons $[43,44]$. This finding is in agreement with other reported studies that have used pedometer to measure level of physical activities based o steps count $[45,46]$. However, we also argued that the pedometer may not be effective for older persons with slow walking speed and shuffling gait. As reported in a previous study, pedometers were less accurate in persons who "shuffle" their feet, such as elders in nursing home residents or those who lean on a walker [47]. Therefore, to minimize the bias and potential for ineffective results, we only include participants with reasonable walking pattern or those without shuffling gait.

In addition, the findings of this current study suggest that an exercise program combined with behavioral program is effective not only in increasing walking distance but also in improving older person belief and knowledge about the importance of exercising and physical activity. This can be seen among the participants in EBG who received additional behavioral program, as they were introduced with specific modelling, goal-setting, self-monitoring, and performance feedback. Thus, this supports the importance of the provisions of knowledge and skills related to exercise and physical activity in order to facilitate a behaviour change.
Generally speaking, older persons with a high level of self-efficacy may have better participation and engagement in a long term physical activity and exercise. In this study, participants who received exercise and behavioral program (EBG) have high levels of exercise self-efficacy, specifically at the 24th week. Their level of confidence in performing an exercise kept increasing as they want to achieve their short term and long term goals. Participants revealed that their goals are most useful when they are specific, realistic, and important for them. When setting their own specific goal, (e.g., walking $3 \mathrm{~km}$ for this week and increase $2 \mathrm{~km}$ for the following weeks), researchers continuously reminded participants to see and review the list of goals regularly. This might influence the inner motivation needed in order for participants to keep performing physical activity or exercise. This view is consistent with a previous study that had found a strong association between exercise self-efficacy and level of exercise participation among older African-Americans [48]. Self-efficacy has the benefit of significantly predicting exercise behavior at follow-up when biological and behavioral influences were controlled [48]. Therefore, in this behavioral program, we included the module of goal setting, the barriers and motivators to exercising, and other parameters as well as a module regarding the risk and benefits of exercise.

The behavioral program also allows the researchers to promote and support exercise as a constructive means of prevention while incorporating it into general health promotion activities. Participants in the EBP were also found to have significantly increased level of self-confidence while performing exercise compared to both EG and CG due to the power of leadership. In fact, the participants in EBG formed their own exercise group after the termination of the behavioral program without any support from the researchers. Exercises were conducted and performed based on the recommendations of the behavioral manual. Each participant showed strong commitment and cooperation especially in reminding each other regarding the type, frequency, and time to exercise. The role of group support may indicate the importance of the partnership factor and can be applied in any kind of situations. For instance, participants in the group can remind their partners of their strengths and how even seemingly small contributions can help in increasing the belief of performing an exercise.

As revealed in the results of this current study, participants in EG and CG also showed an increase in their belief of exercising based on the exercise self-efficacy score. Nonetheless, the ESE scale is a self-reported questionnaire, and this might contribute to bias among the participants. Affuso et al. [49] suggested that self-reported behavioral 
questionnaires may result in subjects intentionally providing inaccurate answers during interview due to pressure or socially acceptable manner. Other studies suggested that the influence on cognitive parts in the behavioral program [5052].

In this study, the interventions were conducted in a group-based setting. We believe that a group-based intervention may yield better results compared to individualized program as it may enhance social support and increase skills in building relationship between members of the group. According to Bartels [53], older persons can gain benefit more from group interaction, when groups are composed of other older persons. However, an individualized program may be more effective for special cases such as older persons with depression [54]. Nevertheless, future study should attempt to establish older person's preference on the type of intervention as each person may have different levels of motivation.

Several limitations were noted in this study, particularly in research involving quasi-experimental design. This design lacks randomization to intervention groups, which characterizes true experiments. Furthermore, this study only focused on Malay ethnic residing in the community that may lead to bias to other races and setting. Future studies should involve diverse ethnicity due to the differences in the cultural belief and behavior.

Lastly, the findings and recommendations from this study are beneficial and crucial as it may increase exercise adherence and self-efficacy among community-dwelling elderly or specifically for those having barriers towards exercise participations. This study has established the combination of physical and psychological approaches with the intention to maximize older persons' psychological resources, namely, exercise adherence and exercise self-efficacy. Successful ageing is not only about the maintenance of health and exercising regularly but also about positive behavior and attitudes towards exercise participation. These lifestyles changes in older people may have beneficial effects on health and longevity, as well as on the enhancement of quality of life.

\section{Conclusion}

As a conclusion, this study suggests that the behavioral program with a pedometer-based feedback may be an effective intervention especially if the aim is to increase the level of physical activity. Interventions such as a combination of goal setting, contract, feedback, consequences, self-monitoring, and prompts in addition to a multicomponent exercise program may be beneficial for older persons residing in the community.

\section{Acknowledgment}

This research project was supported by Grants from the Research Management Institute Universiti Teknologi Mara (600-RMI/DANA 5/3/RIF (511/2012).

\section{References}

[1] E. M. Phillips, J. C. Schneider, and G. R. Mercer, "Motivating elders to initiate and maintain exercise," Archives of Physical Medicine and Rehabilitation, vol. 85, no. 3, pp. S52-S57, 2004.

[2] I. D. Cameron, G. R. Murray, L. D. Gillespie et al., "Interventions for preventing falls in older people in nursing care facilities and hospitals," Cochrane Database of Systematic Reviews, no. 1, Article ID CD005465, 2010.

[3] A. S. Zago, J. Park, N. Fenty-Stewart, E. Kokubun, and M. D. Brown, "Effects of aerobic exercise on the blood pressure, oxidative stress and eNOS gene polymorphism in pre-hypertensive older people," European Journal of Applied Physiology, vol. 110, no. 4, pp. 825-832, 2010.

[4] D. D. F. M. Rocco, L. S. Okuda, R. S. Pinto et al., "Aerobic exercise improves reverse cholesterol transport in cholesteryl ester transfer protein transgenic mice," Lipids, vol. 46, no. 7, pp. 617-625, 2011.

[5] H. Blake, "Physical activity and exercise in the treatment of depression," Frontiers in Psychiatry, vol. 3, article 106, 2012.

[6] K. J. Stewart, "Physical activity and aging," Annals of the New York Academy of Sciences, vol. 1055, pp. 193-206, 2005.

[7] A. B. Irvine, V. A. Gelatt, J. R. Seeley, P. Macfarlane, and J. M. Gau, "Web-based intervention to promote physical activity by sedentary older adults:randomized controlled trial," Journal of Medical Internet Research, vol. 15, no. 2, pp. 1-43, 2013.

[8] W. J. Chodzko-Zajko, D. N. Proctor, M. A. Fiatarone Singh et al., "Exercise and physical activity for older adults," Medicine and Science in Sports and Exercise, vol. 41, no. 7, pp. 1510-1530, 2009.

[9] J. Rice and J. W. L. Keogh, "Power training: can it improve functional performance in older adults? A systematic review," International Journal of Exercise Science, vol. 2, no. 2, pp. 131151, 2009.

[10] R. J. Nied and B. Franklin, "Promoting and prescribing exercise for the elderly," The American Family Physician, vol. 65, no. 3, pp. 419-426, 2002.

[11] N. Gusi, J. C. Adsuar, H. Corzo, B. del Pozo-Cruz, P. R. Olivares, and J. A. Paracca, "Balance training reduces fear of falling and improves dynamic balance and isometric strength in institutionalised older people: a randomised trial," Journal of Physiotherapy, vol. 58, no. 2, pp. 97-104, 2012.

[12] J. Cohen-Mansfield, M. S. Marx, and J. M. Guralnik, "Motivators and barriers to exercise in an older community-dwelling population," Journal of Aging and Physical Activity, vol. 11, no. 2, pp. 242-253, 2003.

[13] M. J. Findorff, J. F. Wyman, and C. R. Gross, "Predictors of longterm exercise adherence in a community-based sample of older women," Journal of Women's Health, vol. 18, no. 11, pp. 1769-1776, 2009.

[14] A. Bandura, Self-Efficacy: The Exercise of Control, W. H. Freeman, New York, NY, USA, 1997.

[15] N. E. Sherwood and R. W. Jeffery, "The behavioral determinants of exercise: implications for physical activity interventions," Annual Review of Nutrition, vol. 20, pp. 21-44, 2000.

[16] M. E. Tinetti and L. Powell, "Fear of falling and low selfefficacy: a cause of dependence in elderly persons," Journals of Gerontology, vol. 48, pp. 35-38, 1993.

[17] E. McAuley, J. Katula, S. L. Mihalko et al., "Mode of physical activity and self-efficacy in older adults: a latent growth curve analysis," Journals of Gerontology B, vol. 54, no. 5, pp. 283-292, 1999. 
[18] P. M. Burbank, D. Reibe, C. A. Padula, and C. Nigg, "Exercise and older adults: changing behavior with the transtheoretical model," Orthopaedic Nursing, vol. 21, no. 4, pp. 51-61, 2002.

[19] A. Fiske, J. L. Wetherell, and M. Gatz, "Depression in older adults," Annual Review of Clinical Psychology, vol. 5, pp. 363389, 2009.

[20] H. Basler, H. Bertalanffy, S. Quint, A. Wilke, and U. Wolf, "TTM-based counselling in physiotherapy does not contribute to an increase of adherence to activity recommendations in older adults with chronic low back pain-a randomised controlled trial," European Journal of Pain, vol. 11, no. 1, pp. 31-37, 2007.

[21] T. T. Huang, L. H. Yang, and C. Y. Liu, "Reducing the fear of falling among community-dwelling elderly adults through cognitive-behavioural strategies and intense Tai Chi exercise: a randomized controlled trial," Journal of Advanced Nursing, vol. 67, no. 5, pp. 961-971, 2011.

[22] M. de Groot, M. Kushnick, T. Doyle et al., "A model of community-based behavioral intervention for depression in diabetes: program ACTIVE," Diabetes Spectrum, vol. 23, no. 1, pp. 18-25, 2010.

[23] S. E. Crouter, P. L. Schneider, M. Karabulut, and D. R. Bassett Jr., "Validity of 10 electronic pedometers for measuring steps, distance, and energy cost," Medicine and Science in Sports and Exercise, vol. 35, no. 8, pp. 1455-1460, 2003.

[24] P. L. Schneider, S. E. Crouter, O. Lukajic, and D. R. Bassett, "Accuracy and reliability of 10 pedometers for measuring steps over a 400-m walk," Medicine and Science in Sports and Exercise, vol. 35, no. 10, pp. 1779-1784, 2003.

[25] P. L. Schneider, S. E. Crouter, and D. R. Bassett, "Pedometer measures of free-living physical activity: comparison of 13 models," Medicine and Science in Sports and Exercise, vol. 36, no. 2, pp. 331-335, 2004.

[26] G. S. Kolt, G. M. Schofield, N. Kerse, N. Garrett, T. Ashton, and A. Patel, "Healthy steps trial: pedometer-based advice and physical activity for low active older adults," Annals of Family Medicine, vol. 10, no. 3, pp. 206-212, 2012.

[27] M. Justine and T. A. Hamid, "Effects on depression and quality of life," Journal of Gerontological Nursing, vol. 36, no. 10, pp. 3241, 2010.

[28] T. Smith, N. Gildeh, and C. Holmes, "The Montreal cognitive assessment: validity and utility in a memory clinic setting," Canadian Journal of Psychiatry, vol. 52, no. 5, pp. 329-332, 2007.

[29] I. Schöllgen, O. Huxhold, and F. Schmiedek, "Emotions and physical health in the second half of life: interindividual differences in age-related trajectories and dynamic associations according to socioeconomic status," Psychology and Aging, vol. 27, no. 2, pp. 338-352, 2012.

[30] I. N. Clark, N. F. Taylor, and F. J. Baker, "Music interventions and physical activity in older adults: a systematic literature review and meta-analysis," Journal of Rehabilitation Medicine, vol. 44, no. 9, pp. 710-719, 2012.

[31] J. O. Prochaska, C. C. DiClemente, and J. C. Norcross, "In search of how people change: applications to addictive behaviors," The American Psychologist, vol. 47, no. 9, pp. 1102-1114, 1992.

[32] M. C. McNulty, J. Johnson, J. L. Poole, and M. Winkle, "Using the transtheoretical model of change to implement home safety modifications with community-dwelling older adults: an exploratory study," Physical and Occupational Therapy in Geriatrics, vol. 21, no. 4, pp. 53-66, 2003.

[33] A. Kirk, F. MacMillan, and N. Webster, "Application of the transtheoretical model to physical activity in older adults with type 2 diabetes and/or cardiovascular disease," Psychology of Sport and Exercise, vol. 11, no. 4, pp. 320-324, 2010.

[34] J. Brug, M. Conner, N. Harré, S. Kremers, S. McKellar, and S. Whitelaw, "The Transtheoretical Model and stages of change: a critique-observations by five commentators on the paper by Adams, J. and White, M. (2004) Why don't stage-based activity promotion interventions work?" Health Education Research, vol. 20, no. 2, pp. 244-258, 2005.

[35] C. J. Jones and D. J. Rose, Strategies to Increase Exercise Adherence in Older Adults, Human Kinetics, Champaign, Ill, USA, 2005.

[36] P. M. Burbank, D. Reibe, C. A. Padula, and C. Nigg, "Exercise and older adults: changing behavior with the transtheoretical model," Orthopaedic Nursing, vol. 21, no. 4, pp. 51-63, 2002.

[37] S. G. Sazlina, C. J. Browning, and S. Shajahan, "Promoting physical activity in sedentary elderly Malays with type 2 diabetes: a protocol for randomised controlled trial," The British Medical Journal, vol. 2, pp. 1-9, 2012.

[38] C. Tudor-Locke and D. R. Bassett Jr., "How many steps/day are enough? Preliminary pedometer indices for public health," Sports Medicine, vol. 34, no. 1, pp. 1-8, 2004.

[39] E. McAuley, J. Katula, S. L. Mihalko et al., "Mode of physical activity and self-efficacy in older adults: a latent growth curve analysis," Journals of Gerontology B, vol. 54, no. 5, pp. P283P292, 1999.

[40] M. Karimlou, F. Zayeri, and M. Salehi, "Psychometric properties of the Persian version of the world health organization's quality of life questionnaire (WHOQOL-100)," Archives of Iranian Medicine, vol. 14, no. 4, pp. 281-287, 2011.

[41] V. S. Conn, J. C. Valentine, and H. M. Cooper, "Interventions to increase physical activity among aging adults: a meta-analysis," Annals of Behavioral Medicine, vol. 24, no. 3, pp. 190-200, 2002.

[42] R. K. Dishman and J. Buckworth, "Increasing physical activity: a quantitative synthesis," Medicine and Science in Sports and Exercise, vol. 28, no. 6, pp. 706-719, 1996.

[43] M. Kang, S. J. Marshall, T. V. Barreira, and J. O. Lee, "Effect of pedometer-based physical activity interventions: a metaanalysis," Research Quarterly for Exercise and Sport, vol. 80, no. 3, pp. 648-655, 2009.

[44] C. G. Kim and Y. S. Lee, "Use of a pedometer to monitor physical activity in older persons: a pilot study," Southern Nursing Research Society, vol. 10, no. 1, 2010.

[45] M. Yamada, S. Mori, S. Nishiguchi et al., "Pedometer-based behavior change program can improve dependency in sedentary older persons: a randomized controlled trial," The Journal of Frailty and Aging, vol. 101, pp. 39-44, 2012.

[46] D. M. Bravata, C. Smith-Spangler, V. Sundaram et al., "Using pedometers to increase physical activity and improve health: a systematic review," The Journal of the American Medical Association, vol. 298, no. 19, pp. 2296-2304, 2007.

[47] E. V. Cyarto, A. M. Myers, and C. Tudor-Locke, "Pedometer accuracy in nursing home and community-dwelling older adults," Medicine and Science in Sports and Exercise, vol. 36, no. 2, pp. 205-209, 2004.

[48] S. L. Cromwell and M. M. Adams, "Exercise, self-efficacy, and exercise behavior in hypertensive older African-Americans," Journal of National Black Nurses' Association, vol. 17, no. 1, pp. 17-21, 2006.

[49] O. Affuso, J. Stevens, D. Catellier et al., "Validity of self-reported leisure-time sedentary behavior in adolescents," Journal of Negative Results in BioMedicine, vol. 10, no. 1, article 2, 2011. 
[50] G. S. Brassington, A. A. Atienza, R. E. Perczek, T. M. DiLorenzo, and A. C. King, "Intervention-related cognitive versus social mediators of exercise adherence in the elderly," The American Journal of Preventive Medicine, vol. 23, no. 2, pp. 80-86, 2002.

[51] M. A. Wieganda, A. K. Troyerbc, C. Gojmeracb, and K. J. Murphybc, "Facilitating change in health-related behaviors and intentions: a randomized controlled trial of a multidimensional memory program for older adults," Aging and Mental Health, vol. 17, no. 7, pp. 806-815, 2013.

[52] D. Cox and H. D'Oyley, "Cognitive-behavioral therapy with older adults," The British Columbia Medical Journal, vol. 53, no. 7, pp. 348-352, 2011.

[53] Canadian Coalition for Seniors' Mental Health, "National guidelines for seniors' mental health," Canadian Geriatric Journal, vol. 9, supplement 2, pp. S36-S70, 2006.

[54] S. J. Bartels, A. R. Dums, T. E. Oxman et al., "Evidence-based practices in geriatric mental health care," Psychiatric Services, vol. 53, no. 11, pp. 1419-1431, 2002. 


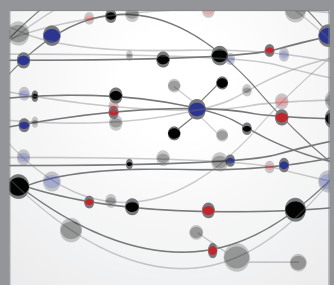

The Scientific World Journal
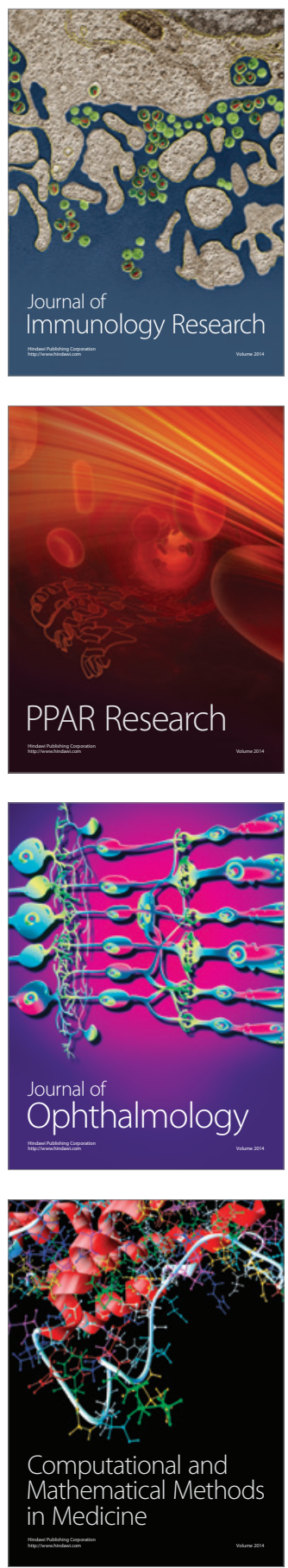

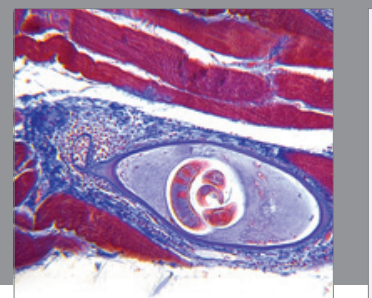

Gastroenterology

Research and Practice
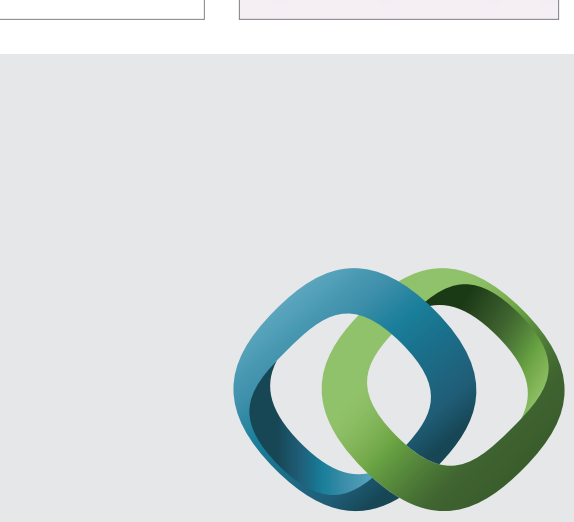

\section{Hindawi}

Submit your manuscripts at

http://www.hindawi.com
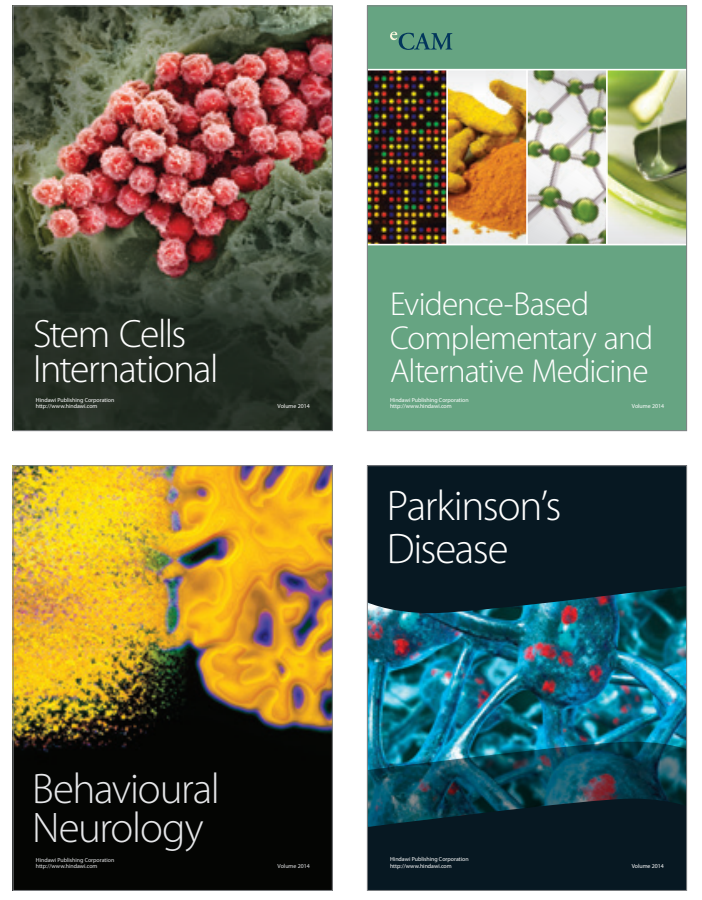
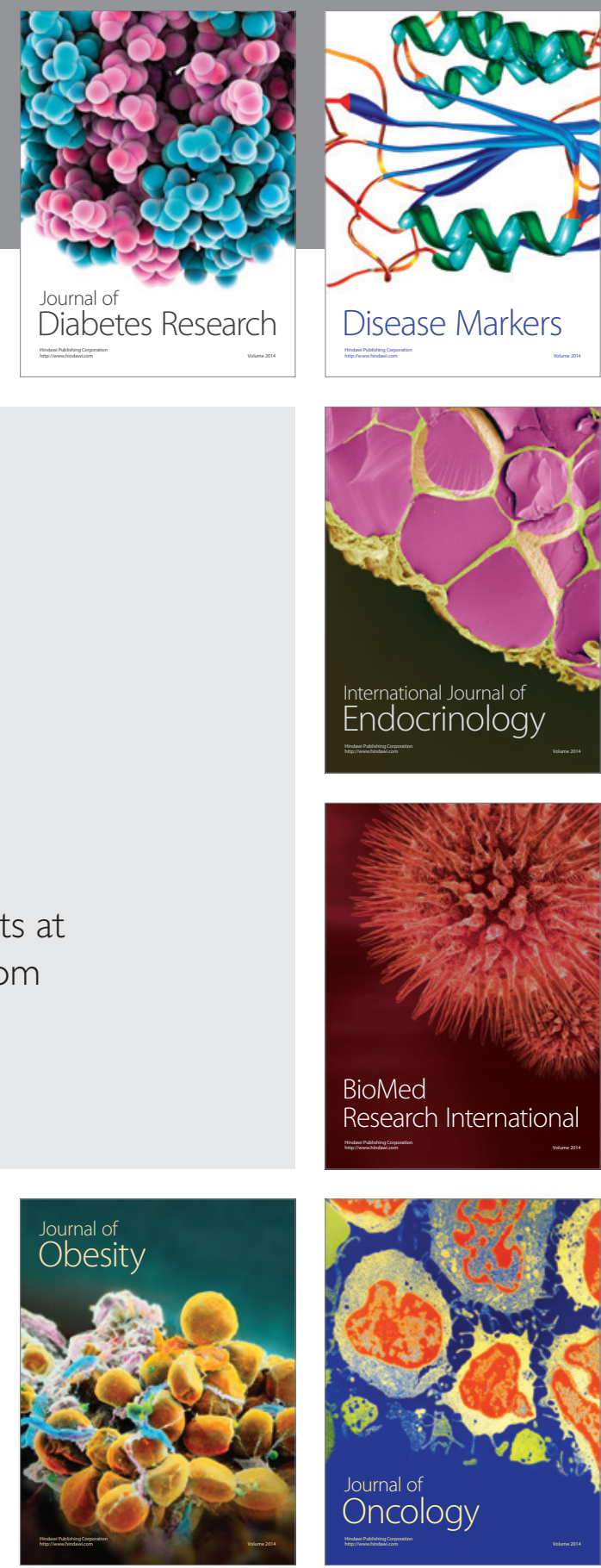

Disease Markers
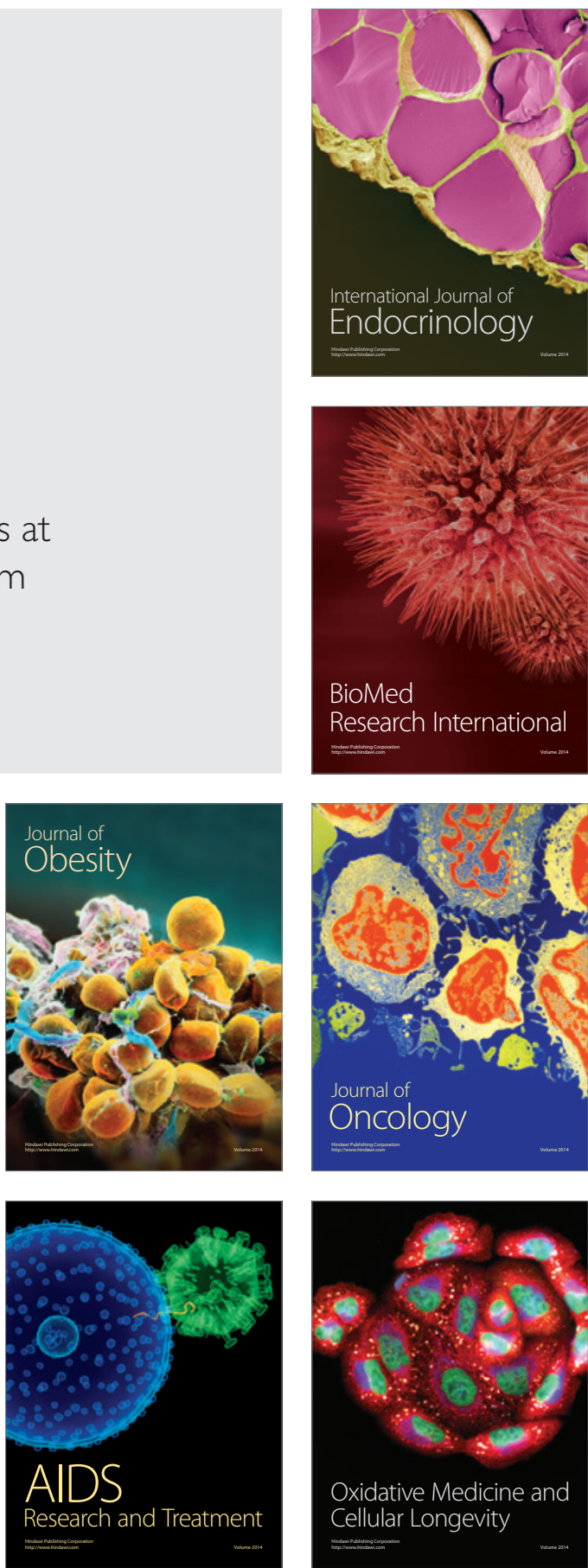